\title{
RELATIVE CHERN-LASHOF THEOREMS
}

\author{
NATHANIEL GROSSMAN
}

We assign to each compact connected manifold with boundary smoothly embedded in a Euclidean space a number, the total absolute curvature, which is positive and at least 1 . We prove that the total absolute curvature can equal 1 only when the embedding is onto a closed convex body of a Euclidean subspace of the same dimension. If the total absolute curvature is less than 2 , we show that the manifold is contractible and its boundary is a homology sphere. The manifold is diffeomorphic to a closed disc, if its dimension is at least 6, and it as well as its boundary is simply connected. We obtain restrictions on the topology, if the total absolute curvature is less than $5 / 2$. Finally we consider a surface in three-space, and relate the total absolute curvature to the knottedness of the boundary. There is a suggestion of a new type of "tight" embedding.

\section{Definition of total curvature}

Let $M$ be an $n$-dimensional connected compact manifold with boundary $\partial M$ smoothly embedded into Euclidean $(n+N)$-space $E^{n+N}$. We do not assume $\partial M$ to be connected, but always require it nonempty. We will assign to $M$ a total absolute curvature $\kappa(M)$ which will always have value at least 1 .

Suppose all the cut points of $M$ and of $\partial M$ in $E^{n+N}$ lie at distance from $M$ greater than $\varepsilon$. Let $M_{\varepsilon}$ be the set of points of $E^{n+N}$ at distance $\varepsilon$ from $M$. The unit normal bundle $\nu$ of $M$ is well-defined. We adjoin to $\nu$ those unit vectors normal to $\partial M$ whose inner product with the outer normal of $\partial M$ relative to $M$ is nonnegative, calling the totality of such vectors together with $\nu$ the augmented unit normal bundle of $M$, denoted by $\nu_{+}$. (Cf. the constructions used by White [9], [10].) Then $M_{\varepsilon}=\left\{p+\varepsilon e \mid p \in M\right.$ and $\left.e \in \nu_{+}\right\}$. Our cut point hypothesis implies $M_{\varepsilon}$ is a submanifold of $E^{n+N}$ of class $C^{1}$ and, in fact, of class $C^{\infty}$ except on the set $\Lambda=\{p+\varepsilon e \mid p \in \partial M$ and $e$ is orthogonal to the normal of $\partial M$ relative to $M$ \}. The measure of $\Lambda$ is zero. There is a map $\pi: M_{\varepsilon} \rightarrow M$ given by $\pi(p+\varepsilon e)=p$. Obviously, $M_{\varepsilon}$ is a closed orientable hypersurface of $E^{n+N}$ whose interior is distinguished by containing $M$.

By Gauss' lemma, the exterior normal to $M_{\varepsilon}$ at $p+\varepsilon e$ is $e$. We obtain a

Received August 9, 1971. The preparation of this paper was supported in part by NSF grant No. GP-11476. 
Gauss map $\Gamma: M_{\varepsilon} \rightarrow S^{n+N-1}$ by $\Gamma(p+\varepsilon e)=e$. Since $M_{\varepsilon}$ and $S^{n+N-1}$ have the same dimension, we obtain a scalar function $G(p, e)$, the Lipschitz-Killing curvature, as a Radon-Nikodym derivative by setting $\Gamma^{*} d S^{n+N-1}=G(p, e) d M_{\varepsilon}$, unless $p+\varepsilon e \in \Lambda$ when we set $G(p, e)=0$. Now we define the total absolute curvature of $M$ by

$$
\begin{aligned}
\kappa(M)= & \frac{1}{c_{n+N-1}} \int_{\text {Int } M} \int_{\pi^{-1}(p)}|G(p, e)| d S_{p}^{N-1} d M \\
& +\frac{1}{c_{n+N-1}} \int_{\partial M} \int_{\pi^{-1}(p)}|G(p, e)| d H_{p}^{N} d \partial M .
\end{aligned}
$$

In this formula, $S_{p}^{N-1}=\pi^{-1}(p)$ is an $(N-1)$-sphere of radius $\varepsilon$ if $p \in \operatorname{Int} M$, $H_{p}^{N}=\pi^{-1}(p)$ is a hemi- $N$-sphere of radius $\varepsilon$ if $p \in \partial M, d W$ denotes the Riemannian volume element of a Riemannian manifold $W$, and the quantity $c_{n+N-1}$ is the volume of the unit $S^{n+N-1}$ in $E^{n+N}$. Our definition is in analogy with, but not in strict correspondence with, the definition of total absolute curvature given by Chern and Lashof [3] for the case of a compact manifold without boundary.

Under the influence of [4] it has become customary to normalize the total absolute curvature defined by Chern and Lashof by dividing it by the volume of an appropriate unit sphere. If $L$ is a compact $n$-manifold without boundary embedded in $E^{n+N}$, we denote its total absolute curvature in the sense of Chern and Lashof (suitably normalized) by $\tau(L)$. Then our total absolute curvature is connected with that of Chern and Lashof by the relation $2 \chi(M)=\tau\left(M_{\varepsilon}\right)$.

B. Y. Chen [2] has defined a total absolute curvature function $T A$ for embeddings of manifolds with boundary. It is given, in fact, by the first summand of our definition, but his definition does not seem capable of yielding theorems. such as we have found.

A second form for $\kappa(M)$ is useful. As a hypersurface in $E^{n+N}, M_{\varepsilon}$ has principal curvatures $\lambda_{1}, \cdots, \lambda_{n+N-1}$ at $p+\varepsilon e$ relative to the exterior normal $e$, except on the null set $\Lambda$. Then

$$
\kappa(M)=\frac{1}{c_{n+N-1}} \int_{M_{\varepsilon}}\left|\lambda_{1} \cdots \lambda_{n+N-1}\right| d M_{\varepsilon} .
$$

A dilation of $E^{n+N}$ with factor $\delta$ takes $M_{\varepsilon}$ onto a manifold where each $\lambda$ is replaced by $\lambda / \delta$, and $d M_{\varepsilon}$ by $\delta^{n+N-1} d M_{\varepsilon}$. The cut locus moves to distance from $M$ greater than $\varepsilon \delta$, and the integral defining $\kappa(M)$ remains invariant: it is positive and homogeneous of degree zero. By choosing $\delta$ sufficiently large, say $\delta>2 / \varepsilon$, we can insure that the cut locus lies at distance from $M$ greater than 1 . It is therefore no loss of generality to assume from this point on that $\varepsilon=1$ and to deal with $M_{1}$. 
There is yet another form for $\kappa(M)$ which is useful. Given any $e \in S^{n+N-1}$, there is a $p \in M_{1}$ with $\Gamma(p+e)=e$, so that $\Gamma$ is surjective. (We may use the standard argument of "bringing a plane in from infinity", perhaps first used by $\mathrm{H}$. Hopf.) If we denote by $m(e)$ the number of points in $\Gamma^{-1}(e) \subset M_{1}$ (finite for almost all $e$ by Sard's theorem) well-known arguments [3] lead to a third expression for the total absolute curvature:

$$
\kappa(M)=\frac{1}{c_{n+N-1}} \int_{S^{n+N-1}} m d S^{n+N-1} .
$$

\section{Minimum total absolute curvature}

We have just observed that $m(e) \geq 1$ for all $e \in S^{n+N-1}$. Therefore $\kappa(M) \geq 1$ always.

Theorem 1. $\kappa(M)=1$ if and only if there is an $E^{n}$ in $E^{n+N}$ into which $M$ is embedded as a closed convex body.

Proof. Suppose $\kappa(M)=1$. Then $\tau\left(M_{1}\right)=2$, so $M_{1}$ is a convex hypersurface in $E^{n+N}$ by [3, Theorem 4]. Of course we can also make the same statement about each $M_{\varepsilon}, 0<\varepsilon<1$. Because the open sets Int $M_{\varepsilon}$ are also convex and

$$
M=\bigcap_{0<\varepsilon<1} \operatorname{Int} M_{\varepsilon},
$$

we find that $M$ is a convex subset of $E^{n+N}$. The conclusion of Theorem 1 follows easily by a dimensional argument.

The following argument also leads to the conclusion starting from only the convexity of $M_{1}$.

Let $p \in \operatorname{Int} M, e \in \nu_{p}$. Denote the principal curvatures of $M$ at $p$ in the direction $e$ by $\mu_{1}(e), \cdots, \mu_{n}(e)$. An easy calculation shows the principal curvatures of $M_{1}$ at $p+e$ in the direction $e$ to be

$$
\lambda_{i}(p+e)= \begin{cases}\mu_{i}(e)\left[1+\mu_{i}(e)\right]^{-1}, & 1 \leq i \leq n \\ 1, & n+1 \leq i \leq n+N-1 .\end{cases}
$$

Since $M_{1}$ is convex, $\lambda_{i}(p+e) \geq 0$, entailing $\mu_{i}(e) \geq 0$, for $1 \leq i \leq n$. Because $\mu_{i}(-e)=-\mu_{i}(e)$, the principal curvatures of $M_{1}$ at $p-e$ in the direction of the outer normal $-e$ are

$$
\lambda_{i}(p-e)= \begin{cases}-\mu_{i}(e)\left[1-\mu_{i}(e)\right]^{-1}, & 1 \leq i \leq n \\ 1, & n+1 \leq i \leq n+N-1 .\end{cases}
$$

The convexity of $M_{1}$ entails $-\mu_{i}(e) \geq 0$ for $1 \leq i \leq n$. (We have twice used the cut locus hypothesis in the form $\left|\mu_{i}(e)\right|<1$.) Therefore $\mu_{i}(e)=0$ for all 
$e \in \nu$ and $1 \leq i \leq n$, so $M$ is totally geodesic. We conclude $M$ lies in some $E^{n}$ of $E^{n+N}$ with Int $M$ open in $E^{n}$ and $\partial M$ a hypersurface of $E^{n}$.

Since $M_{1}$ is convex in $E^{n}, M_{2}=M_{1} \cap E^{n}$ is convex in $E^{n}$. Since $M_{2}$ is the outer parallel surface to $\partial M$ at distance 1 , it is easy to see that $\partial M$ is convex (and, of course, connected).

Conversely, if $M$ is a closed convex body in an $E^{n}$ of $E^{n+N}$, it is quite easy to show $m(e)=1$ except on a set of measure zero in $S^{n+N-1}$, so $\kappa(M)=1$.

\section{Total absolute curvature close to minimum}

Let $L$ be a submanifold of $E^{n+N}$, and let $\beta(L)$ denote the sum of the rational Betti numbers of $L$. It is well-known [4] that $\tau(L) \geq \beta(L)$. We have therefore the following lemma.

Lemma 1. $\kappa(M) \geq \frac{1}{2} \beta\left(M_{1}\right)$.

Lemma 2. Let $L$ be a closed hypersurface of a Euclidean space with $\tau(L)<4$. Then $L$ is diffeomorphic to a sphere.

Proof. Because $\tau(L)<4$, there is a smooth real-valued function on $L$ with less than 4 critical points, all nondegenerate. If the function has two critical points, then $L$ is homeomorphic to a sphere. It follows that $L$ is diffeomorphic to the standard sphere: if $\operatorname{dim} L \leq 3$, by classical results; if $\operatorname{dim} L=4$, by a theorem of Cerf [1]; if $\operatorname{dim} L \geq 5$, because $L$ embeds as a hypersurface. It cannot happen that the function has three critical points, for then $L$ would be a manifold like a projective plane, studied by Eells and Kuiper [5], and no such manifold embeds as a hypersurface into a Euclidean space.

Lemma 3. Let $\kappa(M)<2$. Then $M$ is contractible.

Proof. We are assuming, in fact, that $\tau\left(M_{1}\right)<4$, so $M_{1}$ is $\left(C^{1}-\right)$ diffeomorphic to a hypersphere in $E^{n+N}$ by Lemma 2 . The complement of $M_{1}$ has two open components, the inner component $J_{0}$ containing $M$. Clearly $M$ is a strong deformation retract of $J_{0}$. Since $M_{1}$ is of class $C^{1}$, one form of the differentiable Schoenflies theorem [8, Theorem 3.1] shows that $J_{0}$ is contractible to a point. It follows that $M$ itself is contractible.

Theorem 2. Let $\kappa(M)<2$. Then $\partial M$ is an integral homology sphere (and, in particular, has one component if $n \geq 2$ ).

Proof. According to the Poincaré duality theorem [6, Theorem 7.5], $H_{k}(M, \partial M)$ is isomorphic to $H^{n-k}(M)$ for all $k$. By Lemma 3, $M$ is contractible. The conclusion follows from elementary homology arguments. q.e.d.

The low dimensional cases of Theorem 2 deserve some comment. The case $n=1$ is trivial, $M$ being a closed interval. In case $n=2, \partial M$ must be $S^{1}$ and $M$ the 2-disc $D^{2}$, since we know from the ancient homology theory of surfaces that no other surface bounded by $S^{1}$ can have the homology of a point. The nature of $M$ in dimension 3 seems to be tied up with the Poincaré conjecture and in dimension 4 with the "4-Disc conjecture" [6, p. 113]. 
There are various ways of characterizing the closed $n$-disc $D^{n}$ amongst manifolds with boundary, starting from the $h$-cobordism theorem. Here is one example [6, p. 108].

Proposition A. Suppose $W^{n}$ is a compact simply connected smooth $n$-manifold, $n \geq 6$, with a simply connected boundary. Then the following four assertions are equivalent:

(1) $W^{n}$ is diffeomorphic to $D^{n}$,

(2) $W^{n}$ is homeomorphic to $D^{n}$,

(3) $W^{n}$ is contractible,

(4) $W^{n}$ has the integral homology of a point.

If we are willing to make simple topological assumptions on $M$ and $\partial M$, we may invoke the $h$-cobordism theorem.

Theorem 3. Suppose $M$ is a compact simply connected $n$-manifold, $n \geq 6$, with simply connected boundary, smoothly embedded in a Euclidean space. Suppose $k(M)<2$. Then $M$ is diffeomorphic to $D^{n}$.

Proof. By Lemma 3, $M$ is contractible, so fulfills assertion (3) of Proposition $\mathrm{A}$. The conclusion of the theorem is the equivalent assertion (1).

\section{Stretching the limits a bit more}

If we make no hypotheses on the homology of $M_{1}$, we can obtain a relation amongst the Euler characteristics of $M_{1}, M$, and $\partial M$. There is a Mayer-Vietoris decomposition $M_{1}=X_{1} \cup X_{2}$, where $X_{1}=\{p+e \mid p \in M, e \in \nu\}$ and $X_{2}=$ $\left\{\pi^{-1}(p) \mid p \in \partial M\right\}$. The subspaces $X_{1}, X_{2}$, and $X_{1} \cap X_{2}$ are fiber bundles whose base and fiber are, respectively, $M$ and $S^{N-1}, \partial M$ and $D^{N}$, and $\partial M$ and $S^{N-1}$. The Mayer-Vietoris relation for Euler characteristics gives

$$
\chi\left(M_{1}\right)+\chi\left(X_{1} \cap X_{2}\right)=\chi\left(X_{1}\right)+\chi\left(X_{2}\right) .
$$

Using the product relation for Euler characteristics in a bundle, we obtain

$$
\chi\left(M_{1}\right)+(-1)^{N-1} \chi(\partial M)=\left[1+(-1)^{N-1}\right] \chi(M) .
$$

Because the Euler characteristic of a closed odd-dimensional manifold is zero, we have the following relations.

Lemma 4. (a) Suppose $N$ even. Then $\chi\left(M_{1}\right)=\chi(\partial M)$. (b) Suppose $N$ odd and $n$ even. Then $\chi\left(M_{1}\right)=2 \chi(M)$.

The next result is a simple consequence of Lemmas 4,1 , and the obvious inequality $|\chi(L)| \leq \beta(L)$.

Theorem 4. Suppose $\kappa(M) \leq r$. Then

(a) $|\chi(\partial M)| \leq 2 r$ if $N$ is even,

(b) $|\chi(M)| \leq r$ if $N$ is odd and $n$ is even.

Now we stretch the bounds on $\kappa(M)$ a little past the limit allowed in Theorems 2 and 3, and sharpen the conclusion of Theorem 4. 
Theorem 5. Suppose $\kappa(M)<5 / 2$. (a) If $N$ is even, then $\chi(\partial M)=0,2$, or 4. (b) If $N$ is odd and $n$ is even, then $\chi(M)=0,1$, or 2 .

Proof. Since $\kappa(M)<5 / 2$, we have $\tau\left(M_{1}\right)<5$, so that $\beta\left(M_{1}\right)=2,3$, or 4 . We may exclude $\beta\left(M_{1}\right)=3$ for reasons stated in the proof of Lemma 2 . If $\beta\left(M_{1}\right)=2$, then $\chi\left(M_{1}\right)=0$ or 2 .

We need only analyze $\beta\left(M_{1}\right)=4$ further. The Betti numbers of $M_{1}$ in dimensions 0 and $n+N-1$ are 1 , the other Betti numbers summing to 2 if $\beta\left(M_{1}\right)=4$. By Poincaré duality, either (i) $n+N-1$ is even and the Betti number in dimension $\frac{1}{2}(n+N-1)$ is 2 , all others being 0 , or (ii) there is a $d<n+N-1-d$ with Betti numbers 1 in dimensions $d$ and $n+N-1-d$ and all others 0 . In case (i), $\chi\left(M_{1}\right)=0$ or 4 according as $n+N=4 k+3$ or $4 k+1$. In case (ii), $\chi\left(M_{1}\right)=0,2$, or 4 according to various possibilities for the parities of $d$ and $n+N$. The conclusions (a) and (b) follow using Lemma 4.

\section{Surfaces in 3-space and knotted curves}

Let $M$ be an orientable surface in $E^{3}$ with boundary curve $C$ oriented so that the forward tangent to $C$ together with the inner tangent normal form a positively oriented frame. (It was proved four decades ago by Frankl and Pontryagin and by Seifert that any curve $C$ in $E^{3}$ is the boundary of an orientable surface. The genus of $C$ is the smallest genus of such a surface spanning $C$.) Of the two integrals in the first definition of $k(M)$, the first is seen to be

$$
\frac{1}{2 \pi} \int_{M}|K| d M,
$$

where $K$ is the Gauss curvature function on $M$. The second integral can be reduced (cf. [10]) to

$$
\frac{1}{2 \pi} \int_{C}\left(k_{g}^{2}+k_{n}^{2}\right)^{1 / 2} d s=\frac{1}{2 \pi} \int_{C} k d s,
$$

where $k_{g}$ and $k_{n}$ are respectively the geodesic and normal curvatures of $C$ with respect to $M, s$ is the arc length of $C$, and $k$ is the curvature of $C$ in $E^{3}$.

We can now give the following specialization of Theorems 2, 3, 4 and 5 .

Theorem 6. Let $M$ be an orientable surface in $E^{3}$ with connected boundary curve C. Suppose

$$
\iint_{M}|K| d M+\int_{C} k d s \leq 2 \pi r .
$$

(a) If $r=1$, then $M$ is a planar disc with convex boundary. 
(b) If $r<5 / 2$, then $M$ is diffeomorphic to a closed disc, and $C$ is unknotted.

(c) In general $|\chi(M)| \leq r$, and the genus of $C$ is at most $\frac{1}{2}(1+r)$.

Proof. Only (b) does not follow directly from the previous theorems. The assumption $r<5 / 2$ implies $\beta\left(M_{1}\right)=2$ or 4 . Since $M_{1}$ is a closed orientable surface, $M_{1}$ is the sphere or the torus. But $M_{1}$ is diffeomorphic to the double of $M$, so $\chi(M)=\frac{1}{2} \chi\left(M_{1}\right)=0$ or 1 . Since $M$ is orientable with connected boundary, $\chi(M) \neq 0$ and thus $\chi(M)=1$. Therefore $M$ is a disc. q.e.d.

We note that the conclusion (c) of Theorem 6 follows directly from the Gauss-Bonnet theorem.

A direct proof of conclusion (a) of Theorem 6 can be given as follows. By Fenchel's inequality, $\int_{C} k d s \geq 2 \pi$, so $\iint_{M}|K| d M=0$, whence $K \equiv 0$ on $M$. Hence $M$ is flat. But $\int_{C} k d s=2 \pi$ implies that $C$ is a convex plane curve, so $M$ is planar and a disc.

Milnor [7] showed that $\int_{C} k d s<4 \pi$ implies that $C$ is unknotted (that is, $C$ bounds an embedded disc). The conclusion (b) of Theorem 6 may be interpreted as a condition that $C$ be an unknotted curve.

Corollary 1. If $C$ is knotted and bounds an orientable surface $M$ in $E^{3}$, then $\kappa(M) \geq 5 / 2$.

What if $C$ bounds a nonorientable surface $M$ in $E^{3}$ ? We obtain a surface $M_{1}$ as before, and again $M_{1}$ is closed and orientable. We define $\kappa(M)=\frac{1}{2} \tau\left(M_{1}\right)$, just as in the orientable case. The relation $\chi\left(M_{1}\right)=2 \chi(M)$ remains true, for the set $X_{1}$ in the Mayer-Vietoris decomposion of $M_{1}$, no longer a product, is fibered over $M$ with fiber consisting of two points. We obtain this corollary to Theorem 6.

Corollary 2. Let the curve $C$ in $E^{3}$ bound a nonorientable surface $M$ with $\kappa(M)<5 / 2$. Then $M$ is a Möbius band.

In conclusion, we remark that our total absolute curvature $\kappa$ suggests a new tightness concept for submanifolds of Euclidean spaces. If $L$ is a submanifold of a Euclidean space, we may consider all oriented manifolds embedded into that space with $L$ as boundary. It is natural to ask whether one of these manifolds has the minimum possible total absolute curvature. If such a manifold with boundary $L$ exists, we can call $L$ "tightly embedded" (in quotation marks to distinguish the notion from the tight embeddings used by N. H. Kuiper and others). Such a "tightly embedded" $L$ must have its $L_{1}$ tightly embedded in Kuiper's sense. To classify the "tightly embedded" submanifolds, one would first have to classify all tightly embedded hypersurfaces. So far as we are aware, this has not been done. Given this classification, those tightly embedded 
hypersurfaces could be investigated to see which of them arise as parallel surfaces to a manifold with boundary.

\section{Bibliography}

[1] J. Cerf, La nullité du groupe $\Gamma_{4}$, Séminaire H. Cartan, Paris, 1962-63, Exps. 8, 9, $10,20,21$.

[2] B. Y. Chen, On the total absolute curvature of manifolds immersed in Riemannian manifolds. III, Kōdai Math. Sem. Rep. 22 (1970) 385-400.

[ 3 ] S. S. Chern \& R. K. Lashof, On the total curvature of immersed manifolds, Amer. J. Math. 79 (1957) 306-313.

[4] - On the total curvature of immersed manifolds. II, Michigan Math. J. 5 (1958) 5-12.

[5] J. Eells \& N. H. Kiuper, Manifolds which are like projective planes, Inst. Hautes Études Sci. Publ. Math. No. 14 (1962) 5-46.

[6] J. Milnor, Lectures on the h-corbordism theorem, Math. Notes, Princeton University Press, Princeton, 1965.

[ 7 ] - On the total curvature of knots, Ann. of Math. 52 (1950) 248-257.

[ 8 ] M. Morse, Schoenflies problems, Fund. Math. 50 (1962) 319-332.

[9] J. H. White, Self-linking and the Gauss integral in higher dimensions, Amer. J. Math. 91 (1969) 693-728.

[10] - Some differential invariants of submanifolds of Euclidean space, J. Differential Geometry 4 (1970) 207-223.

University of California, Los Angeles 